\title{
El miedo a la paz ${ }^{1}$
}

\section{Fear of peace}

\author{
Juan Camilo Ruiz Salazar²
}

\section{Resumen}

Este escrito busca realizar una mirada a la construcción del miedo a la paz desde dos nociones que se consideran relevantes: tiempo y espacio. El tiempo desde la construcción del pasado, presente y futuro, el espacio desde el territorio. Para este fin se realizan dos aproximaciones, una de orden reflexivo y la otra a partir de expresiones relacionadas con al resultado de los diálogos de paz entre el gobierno de Juan Manuel Santos y las FARC-EP. Este recorrido se mantiene en el pasado y en el presente, para entonces llegar al futuro, donde a partir de la explicación del líder de la oposición al proceso de paz, Álvaro Uribe Vélez, se materializa el miedo a la paz, para apostar por formas otras en que es posible narrar el pasado para construir otros futuros.

Palabras clave: Miedo; Paz; Conflicto.

\begin{abstract}
This paper seeks to look at the construction of the fear of peace from two notions that are considered relevant: time and space. Time from the construction of the past, present and future, space from the territory. To this end, two approaches are made, one of a reflexive order and the other based on expressions related to the result of the peace dialogues between the government of Juan Manuel Santos and the FARC-EP. This route is maintained in the past and in the present, to then reach the future, where from the explanation of the leader of the opposition to the peace process, Álvaro Uribe Vélez, the fear of peace materializes, to bet on other ways in which it is possible to narrate the past to build other futures.
\end{abstract}

Keywords: Fear; Peace; Conflict.

\section{Introducción}

El miedo se relaciona con las lógicas del tiempo, en especial las posibilidades de lo que podría ser el futuro. Es decir, se ubica en la existencia o producción social de la idea del futuro, la cual se encuentra adelante, en movimiento constante, a partir de las acciones del hombre, donde se materializa la latencia de lo que podrá ser, y en esa latencia se

\footnotetext{
1 Trabajo recibido el 21/08/2019. Aceptado el 17/10/2019.

${ }^{2}$ Universidad Central de Colombia. Contacto: juanruizsalazar@gmail.com
} 
encuentra el miedo con su mayor posibilidad de producir sentido en las prácticas del hoy.

El tiempo como producción humana se puede reconocer en su triple dimensión, el pasado, el presente y el futuro, estos se relacionan con un espacio, es decir, es imposible separar la idea del tiempo con la del espacio (Wallerstein, 1998).

El espacio en el cual se desarrolla esta reflexión sobre el miedo a la paz es Colombia, un territorio nacional del norte de Suramérica, el cual cuenta con un tiempo, es decir, un pasado, un presente y, tal vez, un futuro. De igual forma al reconocimiento de un espacio, es preciso poder establecer las coordenadas del tiempo, pues, Colombia como espacio no siempre existió y por ese mismo motivo se puede poner en duda su futuro. El tiempo pasado, en este caso se establece en el siglo XX, el presente en la segunda década del siglo XXI y el futuro las décadas por venir.

El miedo funciona en relación con el tiempo, de allí su relación como dispositivo de poder, pues se arraiga en los futuros posibles, pero para poder proyectarse es preciso reconocerse en el pasado, se trata principalmente de la historia, de la oficial, la de los vencedores. Esta idea del pasado y la historia se encuentra en las ciencias sociales como un factor preponderante para la comprensión de los fenómenos sociales (Wallerstein, 1996), de hecho, la presencia de relatos históricos escritos permitió diferenciar un pueblo civilizado de uno bárbaro, es esta la potencia de la historia.

La triple dimensión del tiempo en el espacio funciona como una proyección, hacia el futuro posible, donde el pasado (atrás) ${ }^{3}$ con sus cargas históricas permiten comprender el presente para orientar las acciones del futuro (adelante) mediante prácticas sociales, políticas y económicas. Es por esta forma, que los relatos del pasado construidos y compartidos socialmente son en muchas ocasiones un factor para comprender los miedos que pueden cristalizarse en el futuro y que funcionan como latencias en el presente orientando las prácticas sociales.

\footnotetext{
${ }^{3}$ Se trata de la relación con los procesos metafóricos orientacionales, que permiten brindar coordenadas de espacio a las formas en que significamos mediante metáforas el mundo (Lakoff \& Johnson, 2017).
} 


\section{El pasado}

El pasado colombiano cuenta con un elemento profundo de reflexión, se trata del conflicto el cual se puede presentar a partir de dos esferas de análisis, una material y otra inmaterial. La primera, causa de unos procesos históricos de la larga duración que llevan a la conformación y fundamentación de la segunda (PNUD, 2003). Así las cosas, es preciso iniciar reconociendo no solo estos dos aspectos sino también, que el conflicto es necesario y deseado en nuestras sociedades (Gutiérrez, 2017), pues es en el conflicto donde se puede reconocer el atrás, es decir, el pasado actualizándose en el presente.

La principal característica del conflicto se refugia en la desigualdad, donde un grupo de sujetos históricamente se han apropiado de las mayores posibilidades materiales de lograr sus ejercicios de crecimiento (Osses, 2017), se trata por lo tanto de una hegemonía (Laclau, 2014), que cuenta con la acumulación material necesaria para garantizar su desarrollo. Esto ha llevado a que la desigualdad históricamente se encuentre reflejada en una serie de poblaciones en especial, reconocidas bajo las categorías de género, clase, raza y orientación sexual (Castro Gómez, 2017), siendo así estos grupos poblacionales los que se encuentran con las menores posibilidades materiales de garantizar su desarrollo.

La acumulación de recursos de diversa índole, no solo económica, permite la producción de lo que Pablo Gutiérrez (2017) reconoce como la concepción paranoide de la realidad, retomando el trabajo de Estanislao Zuleta (2015); el cual se configura cuando a partir de la acumulación de recursos por parte de un grupo social, se establecen dos grupos de interacción en la sociedad, un nosotros y un ellos, donde el ellos siempre será un sujeto que actúa en términos de «maldad» frente al nosotros.

Lo anterior permite comprender la separación u organización del espacio (país) a partir de un enemigo común reconocido en el ellos, así, se tratará de una relación donde siempre podemos construir de forma organizada o necesaria el enemigo que nos constriñe en términos de nuestra posibilidad de ser y hacer (Restrepo, 2017). El conflicto por lo tanto no se encuentra principalmente en la acumulación de las posibilidades materiales de ser o hacer por parte de un grupo específico de la población, sino en la construcción, de ese otro como un enemigo que sólo puede buscar el daño o la maldad en las condiciones que ya el nosotros posee. Es decir, el que acumula las posibilidades de desarrollo encuentra en aquellos que quieren otras posibilidades de desarrollo la mal- 
dad de quien quiere quitarle algo que por derecho histórico ${ }^{4}$ le pertenece, mientras que quien busca ampliar sus posibilidades encuentra en aquel que ya las posee, una maldad profunda en el no querer compartir un mínimo de sus posibilidades. Se trata por lo tanto de una negación constante de las posibilidades de los sujetos de diversos lugares por impedir que el otro pueda ser o hacer (Castro Gómez, 2017).

Ahora bien, este tipo de conflicto del orden paranoide, produce una serie de desacuerdos profundos frente a la organización social del país, donde la posibilidad de dialogo o resolución del conflicto se anula, pues el otros ha sido construido como un riesgo profundo, éste riesgo se basa en la desconfianza sobre los intereses del otro y por los procesos históricos (Gómez Restrepo, 2017). La ausencia de confianza entre las partes lleva a imposibilitar las formas de resolución del conflicto que no requieran del sometimiento físico o simbólico del otro, pues es imposible dialogar con el que actúa desde la maldad, por lo tanto uno de los principales efectos del conflicto es el desconocimiento de la posibilidad del bien común en las acciones de aquellos otros (López, 2017).

Acorde con lo anterior, el conflicto no solo se materializa en la acumulación de posibilidades de desarrollo por grupos de sujetos en el espacio colombiano, sino que además se materializa a su vez en acciones de sometimiento, anulación y muerte, que terminan siendo al parecer las únicas acciones válidas para dialogar con el otros desde el nosotros (Useche, 2017), como si por ejemplo la felicidad solo pudiese ser comprendida desde una perspectiva única que uniforma a todos los agentes sociales colombianos. ${ }^{5}$

En este orden de ideas, el conflicto colombiano desbordando la idea del conflicto armado, se arraiga en la posibilidad de acceso a condiciones mínimas para el desarrollo de los sujetos en el contexto nacional, sin embargo, la lucha constante entre: los ellos y los nosotros, ha logrado la consolidación de un orden político específico en el país, mediante lo que Eduardo Restrepo (2017) reconoce como sujetos pusilánimes, en la

\footnotetext{
${ }^{4}$ Aquí es clave comprender como la historia puede servir para legitimar un lugar de poder para un grupo que posee bienes materiales y simbólicos, así, por ejemplo, la historia sirvió para legitimar el orden establecido por los reyes como encomendados de dios, hoy sirve para legitimar un orden político: la democracia y la existencia de los Estados Nacionales.

${ }^{5}$ La producción de hegemonías totalizantes, conlleva la desaparición de la hegemonía en sí misma, es decir, los procesos de producción hegemónicos requieren de la existencia de lo contra hegemónico para su propia reafirmación y existencia, de lo contrario la posibilidad totalizante borra las diferencias y en ellas la hegemonía en su esencia (Laclau, 2014).
} 
medida que ese mismo conflicto sin resolución garantiza, para ciertos colectivos mayores posibilidades de ser y hacer, mediante la consolidación de sujetos que no luchen o simplemente no levanten la voz frente a aquellas cosas que reconocen como negativas en el panorama nacional, lo anterior en la medida que alzar la voz sería en sí misma un acción de maldad reconocida por el otro, recordando entonces la perspectiva paranoide (Zuleta, 2015) de la realidad en Colombia.

Se trata por lo tanto de la producción profunda de una cultura del sometimiento a partir de una memoria atávica (Useche, 2017), donde los procesos de dominación y en ella, la posibilidad de construir sentido por parte de las y los colombianos, se encuentra enmarcada en un proceso de memoria de muy larga data, que fundamenta la producción del enemigo en cualquier otro que no pertenezca al nosotros.

Esa producción del enemigo, que puede ser también reconocida en la formula de Hobbes, el hombre es lobo para el hombre (Castro Gómez, 2017), de la mano con las servidumbres voluntarias, permite un ejercicio específico del poder en el orden social, donde los grupos de sujetos (nosotros o ellos) entregamos nuestro poder como ciudadanos, a un poderoso que actualiza el poder mediante el miedo a perder lo que tenemos, en cualquiera de los casos, ese miedo actualizado en forma de poder, termina por consolidar la servidumbre voluntaria (Useche, 2017).

Es en ese tipo de relación donde se delega el poder a un poderoso que utiliza como estrategia el sometimiento, a través de la falsa noción de seguridad sobre la posibilidad de perder las cosas o las posibilidades materiales de desarrollarnos, permite la construcción de dos fenómenos en nuestra sociedad, por un lado la idea de la bondad en la obediencia al poderoso sin reflexión, segundo una cultura política de orden autoritario (Restrepo, 2017).

En el primer caso, relacionado con la obediencia, se fortalece la idea de los ciudadanos pusilánimes, en términos de la incapacidad de reflexionar sobre los sentidos del poder y de las acciones (Rorty, 2008), consolidando entonces la legitimidad del poder del poderoso, sin posibilidad alguna de reflexionar en torno a las acciones que este mismo encamina en la garantía del mantenimiento de sus condiciones materiales de desarrollo, aunque estas construyan en quien le delega el poder un otros que atenta frente al nosotros que él o ella representa (Gutiérrez, 2017). Lo anterior se puede reconocer, además, en las formas en que se legitima el uso del poder, donde por ejemplo: si un estudiante le pega a un policía es por lo tanto un criminal, mientras que si el policía le pega al 
estudiante, es porque está instaurando el orden (Martínez, 2017), así entonces queda sin posibilidad la forma de comprender las acciones del otro y sus posibles sentidos en términos de consolidar aquellos que podríamos reconocer como bien común, pues en esta lógica del poder, no es posible reconocer que alguna acción es para beneficiar a muchos, toda acción es simplemente un atentado contra mis intereses y mis necesidades (López, 2017).

En el segundo aspecto, donde se fortalece y fundamenta una cultura política de orden autoritario, los procesos anteriores se materializan, en términos de no poder reconocer al otros, pues siempre estará en condición de dañar al nosotros, por lo tanto requerimos de esa autoridad, del poderoso, ${ }^{6}$ que garantiza el desconocimiento de la diferencia, de la posibilidad, así entonces, la base principal de la cultura política autoritaria en Colombia se materializa en un hecho: la desconfianza del otro, siempre, por ser ellos y no nosotros (Useche, 2017).

Acorde con lo presentado hasta este punto, se cuenta con dos lados del conflicto, el primero de ellos del orden material se trata de las desigualdades sociales, a partir de las cuales, se configura y consolida una forma de cultura política, donde se expresa la inmaterialidad del conflicto, por medio del autoritarismo fortalecido en la desconfianza del otro, incluso en muchas ocasiones del nosotros como comunidad (Torres Carrillo, 2013).

En el espacio Colombia existe en ese conflicto que se desarrolla en líneas anteriores de forma muy marcada, el nosotros y el ellos, el primero encarnado en los ciudadanos que trabajan, de sol a sol, que votan en las elecciones y obedecen los mandatos de sus jefes, pero esos jefes son también parte de ese colectivo, se trata así, de la base económica que permite el desarrollo del país, su crecimiento, se trata de las y los colombianos que trabajan por el país.

A su vez, en esta historia del conflicto, se reconoce un ellos marcado: las guerrillas de izquierda, quienes han torpedeado históricamente las posibilidades de crecimiento económico por la voladura de oleoductos, dinamitando torres eléctricas, secuestrando empresarios, generando inseguridad que no permite la entrada de la inversión extranjera. Esos rasgos son los que se establecen desde el relato más oficial de nuestro

\footnotetext{
${ }^{6}$ Recordando la ansiedad en clave del miedo y política, donde el miedo se tramita por medio de la ansiedad que se puede regular a partir de la existencia de las instituciones sociales como reguladoras de los miedos y garantía del poder en el campo social (Robin, 2009).
} 
pasado, dejando de lado, cómo, por ejemplo, la lucha de grupos armados ilegales como las FARC-EP es la tenencia de tierras en el campo colombiano, donde la reforma agraria se transforma en uno de los principales causantes de la desigualdad, que como observamos es una de las bases materiales del conflicto, sin embargo,la construcción (de las FARC-EP y las demás guerrillas) como el enemigo interno que no permite el desarrollo del espacio colombiano, permitió esa construcción solida de ese ellos, diferente y peligroso.

El pasado, en el espacio colombiano, se encuentra enmarcado en una historia de conflicto armado, con ese ellos maligno, desde donde es posible vincular con ese ellos a cualquier otro colombiano del nosotros que pueda desterrarse de la idea de la servidumbre voluntaria, es decir, pareciera que en este espacio físico colombiano, el nosotros es claramente compuesto por quienes detentan el poder y todos aquellos que se constituyan como servidumbre voluntaria, el ellos, todos aquellos que busquen la autonomía, la libertad y el cambio del orden social establecido por ese nosotros, que ha garantizado históricamente la desigualdad.

Teniendo en cuenta esta construcción del pasado (atrás) bajo la idea de la proyección del tiempo es posible comprender el presente (donde igual el pasado se actualiza). Miremos entonces qué tipo de expresiones se pueden evidenciar en lo que denominamos como presente, la segunda década del siglo XXI.

\section{El presente}

$\mathrm{Al}$ establecer las tres dimensiones del tiempo es posible caer inmediatamente en la idea de la historia como los hechos que toman lugar y dan sentido (Wallerstein, 1998). Esta postura tiene serios problemas, pues se transforma en un compendio de hechos, que en sí mismos no tienen la posibilidad de ofrecernos las evidencias que se requieren para comprender el miedo desde la idea del tiempo.

Lo anterior cobra sentido cuando notamos cómo el pasado se transforma en presente, es decir, se actualiza. El pasado, como forma de pensamiento y reconocimiento del atrás, se encuentra a su vez en un tiempo, siempre, el presente, pues es en el aquí y ahora que reconocemos, producimos y valoramos el pasado para movernos, orientando nuestras prácticas hacia el futuro. 
Así las cosas, la pregunta por el presente se relaciona con las prácticas que se encarnan en el territorio nacional, no como hechos, sino como procesos que encarnan el pasado en la agencia del hoy. Ya reconocemos, entonces, cómo el pasado actualizado en presente relata o justifica un proceso de conflicto, el cual observaremos más adelante (en el futuro), pero en el presente nos ocupa la paz, pues es en ese objetivo de la humanidad que en Colombia se rechazó ${ }^{7}$ un proceso de diálogo y finalización de un conflicto armado por medio del voto popular.

Entonces cabe preguntarse cómo comprender la paz en el hoy y podemos encontrarnos con dos perspectivas diferentes. En la primera de ellas se ha querido instalar el valor o la idea soñada, es decir, todo aquello que desde el pensamiento filosófico podría reconocerse como el camino hacia el bien común y la democracia. En la segunda, las prácticas que se materializan en el diario vivir del país.

En esa primera aproximación, en la paz, se encuentra una solución al conflicto, es decir, una forma de aproximación a otras formas de cultura política, donde los cambios en ésta permiten la configuración de otros conflictos con otras formas de trámite, donde la violencia física o simbólica, manifiesta en la dominación o sometimiento del otro, deja de ser necesaria en cuanto expresión profunda del autoritarismo (Arendt, 1997).

Lo anterior implica aceptar que existen otras formas políticas de relacionamiento, a través de las cuales pueda ser vivido el conflicto no como la negación o puesta en tensión mediante la inseguridad y la desconfianza en la posible pérdida de las condiciones materiales de supervivencia. Esto implica el paso de la política desde el miedo hacia la esperanza, relacionando el cambio entre el pensamiento de Hobbes al de Spinoza (Camps, 2012), como factores fundamentales en la construcción y puja por la legitimidad en el sentido común del pueblo colombiano (Castro Gómez, 2017).

En esta lógica ingresarán por lo tanto cuatro elementos para comprender la paz, se trata de la autonomía, la esperanza, la diversidad y la democracia. Los anteriores como fundamentos que permiten poder salir del círculo que imprime la perspectiva paranoide de la realidad, se tratará de la experiencia de ser plenamente humanos (Osses, 2017).

\footnotetext{
${ }^{7}$ El plebiscito rechazó el resultado del proceso de dialogo, pese a que el gobierno nacional continuó con el proceso y firmó el proceso de paz, que condujo al desarme de las FARC-EP, permitiendo la consolidación del partido Fuerza Alternativa Revolucionaria del Común (FARC), la negativa a su participación política, social y económica en el espacio Colombia sigue siendo un debate de orden nacional.
} 
En un primer lugar se encuentra la autonomía, en la cual se materializa el ejercicio de la libertad, comprendida como el proceso de asumir las responsabilidades de los actos que realiza cada sujeto en el contexto social, comprendiendo que no es posible pensar en el individuo (Wilde, 2016) como unidad separada del contexto y sin interacciones con otros sujetos, por lo tanto, en esta perspectiva de la libertad, y en ella de la autonomía, se gesta la necesidad de los otros, no solo del nosotros (Martínez, 2017).

El anterior punto llevará a la posibilidad de encontrarnos con los otros plenos (Restrepo, 2017), es decir, la necesidad de reconocer a todos los sujetos con los que interactuamos en su plenitud en cuanto seres, con elementos cercanos y otros lejanos, que seguramente nos incomodarán, sin embargo, es en esa posibilidad de la incomodidad donde la autonomía en cuanto sujetos que interactúan y materializan su libertad (Deleuze, 2001), donde el conflicto del orden paranoide comienza a desdibujarse.

Es en la autonomía y la libertad, donde el poder desde el sometimiento pierde su poder en términos de una cultura política de orden autoritario (Nussbaum, 2017), pues permite dejar en evidencia la inoperancia de las servidumbres voluntarias, pues éstas niegan en sí mismas la posibilidad de la autonomía, pues ésta ha sido relegada al poderoso, en este escenario por lo tanto, el poder se transfiere a los denominados débiles, pues los poderosos tienen «pies de barro» (Useche, 2017), en la medida que su poder solo se garantiza en la medida que los sujetos releguen su autonomía, su libertad y en últimas su poder al denominado como poderoso (Robin, 2009).

Se trata por lo tanto de la ruptura de la servidumbre voluntaria, es decir, del poder del poderoso por medio de la dominación. Ahora bien, esta autonomía no se encuentra inscrita en el vacío, sino que se encuentra regulada por las pasiones propone Espinoza (Castro Gómez, 2017), por la pasión de la esperanza (Camps, 2012).

Así entonces ingresa en este escenario la segunda de las categorías clave de la paz, la esperanza en cuanto pasión, de allí que por ejemplo la pregunta por ahora sobre qué construiremos legitimidad política -sobre el miedo a la guerra o la paz, ¿o sobre la esperanza? (Castro Gómez, 2017) - es clave. Ingresa a romper muchos de los establecidos por la cultura política autoritaria que se expresa en el país. La esperanza implica una reorientación del miedo (Deleuze, 2001), pues éste no podrá desaparecer de nuestra vida, siempre estará presente, es parte de la expe- 
riencia humana, está en el cuerpo y culturalmente los expresamos, como el miedo a entregar el cuerpo en un acto con presencia o no de amor (Osses, 2017). Sin embargo el hecho de poder actuar no desde el control ejercido por el miedo sobre nuestros cuerpos, sino desde la esperanza de la posibilidad de interacción con el otro, genera un gran cambio en la cultura, pues de nuevo la autonomía y la libertad son el centro o foco de la posibilidad de ser y hacer, donde el otro ya no representa un riesgo sino una posibilidad (Camps, 2012).

La esperanza, por lo tanto, se transforma aquí en un punto donde es preciso comprender que somos diferentes, que todos pensamos diferente, que eso es cotidiano en una sociedad y, por lo tanto, de hecho es deseable (Gutiérrez, 2017), pues en la medida que existe la diferencia en ella es posible la experiencia del cambio y en él la posibilidad de ser y hacer, reconociendo por lo tanto un equilibrio que dista de la quietud o de la experiencia de un balancín, sino que se trata de un equilibrio como el de la naturaleza, donde cada sujeto, en su diferencia y particularidad, otorga elementos para la existencia del colectivo en cuanto sociedad (Martínez, 2017). Estos elementos nos llevan por lo tanto a la construcción de una autonomía basada en la libertad bajo la esperanza de la oferta del ellos en la construcción del nosotros (Cabra Ayala y Aschner Restrepo, 2017).

En este juego de relaciones se encuentra la diversidad, pues sin ella la posibilidad del otro para el crecimiento del nosotros desaparece. Es por lo tanto relevante reconocer las diferencias que se han construido frente a la clase, el género, la raza y la orientación sexual (Castro Gómez, 2017), pues es en esos otros donde inicialmente se encuentra la posibilidad de construir, a partir del reconocimiento de la esperanza en la interacción con esos otros (Martínez, 2017).

Es preciso reconocer que en la diversidad siempre existirán conflictos, en este caso manifestados por las diferencias en el cómo pensamos y entendemos la vida cotidiana. La paz por lo tanto implica reconocer el conflicto desde un lugar diferente al que se ha descrito en el pasado, la negación plena del ellos, pues en el caso de la paz, el conflicto no se tramita por medio del sometimiento físico o simbólico del otro diferente, sino que se tramita a través de la esperanza del relacionamiento con el otro.

El cuarto elemento de la paz: la democracia, comprendida por ella la mirada más amplia de la misma, donde esa perspectiva estrecha que la relaciona exclusivamente con un proceso de votación queda agotada (Res- 
trepo, 2017), la mirada que implica la comprensión del conflicto y su trámite por medio del reconocimiento del otro plenamente humano con sus diferencias y, en ellas, la posibilidad de cambio y adaptación de la sociedad, mediante la discusión profunda de las apuestas éticas que las diferencias proponen y en ellas la posibilidad de la búsqueda del bien común (Restrepo, 2017).

En este escenario la democracia por lo tanto se desarrolla en un espacio donde se desdibuja el enemigo de la arena política, para consolidar un contrincante con el cual las ideas se encuentran en puja y son los argumentos los que permitirán definir los mejores caminos posibles como sociedad (Castro Gómez, 2017). Lo anterior de hecho lleva a impulsar una interesante tensión por la construcción de nuevas memorias, donde la historia de los vencedores sea reinterpretada, para dar espacio en su formulación a la de los sometidos históricamente, como posibilidad de reconstrucción de una democracia donde al menos «por pensar diferente no seamos asesinados al caminar por la calle» (Castro Gómez, 2017).

Ahora bien, en un recorrido contrario al del conflicto, la paz inicia por un aspecto inmaterial, que se basa en la relación de la autonomía en el ejercicio de la libertad, gobernada por la esperanza que se encuentra en la relación con el otro, vale decir, con la diversidad mediante la discusión y el conflicto tramitado democráticamente (Arendt, 1997). Este ejercicio debería por lo tanto llevar a la resolución de la materialidad del conflicto rastreado en el pasado, el cual como observamos se instala mediante la desigualdad.

Una mirada de la paz, como se ha propuesto, lleva a la imperiosa necesidad de pensar en la equidad, comprendiendo por esta la negación de una distribución igualitaria de los recursos o de las posibilidades materiales para el desarrollo de los sujetos. Por el contrario, se trata de entregar a cada sujeto los elementos que más requiere para su desarrollo en el marco de la sociedad (Gómez Restrepo, 2017).

Es decir, la distribución equitativa de las posibilidades de desarrollo de los sujetos en la sociedad colombiana sería la mejor forma de comprender esa noción de bien común. Esta apuesta es posible en la medida que se cuente con la esperanza de conocer e interactuar con esos otros colombianos y colombianas, para lo cual la autonomía en búsqueda de la diversidad es la forma más apropiada de construir y configurar la democracia.

La posibilidad de ser y hacer, pareciera ser ésta la clave de todo el proceso de la paz como posibilidad de interacción en el conflicto, esa 
posibilidad se materializa en el ser plenamente humanos, desarrollando nuestras posibilidades en cuanto sujetos en el contexto más proclive para el ejercicio de la libertad y la autonomía (Osses, 2017).

Por lo tanto, la paz, no es la ausencia de conflicto, es la ausencia de guerra (Castro Gómez, 2017), donde las posibilidades de los sujetos se encuentran en un escenario en el cual se permite desarrollarse en libertad y autonomía, como sujetos plenos, lejanos de la necesidad de un poder que proteja al hombre del hombre mismo. La paz, será por lo tanto el encuentro entre diferentes en la necesidad de su existencia para completar el equilibrio social, donde además las diferencias se tramitan por medio de la experiencia de la esperanza.

Ahora, el ejercicio del presente desde ese ideal de bien común como la forma de buscar y materializar la paz mediante el reconocimiento de todos, es en medio de todo una solución que dista de las prácticas que se encarnan en el espacio Colombia en el tiempo del presente. Bastaría entonces con señalar que al momento de escribir estas palabras en el país se han asesinado desde la firma con las FARC-EP doscientos dieciséis líderes sociales, muchos de ellos relacionados con la restitución de tierras.

En el presente, desde la presidencia del gobierno de Iván Duque, se plantean cambios importantes al proceso de paz, propuestas sobre la Justicia Espacial para la Paz (JEP), rechazadas por la Corte Constitucional, dejando así en evidencia cómo el camino de reconocer y actualizar el pasado, donde el ellos que se integra a la vida nacional, jamás podrá ser un nosotros, ese ellos, sigue siendo la mejor forma de actualizar el presente y proponer un futuro, de conflicto sin paz como se trató de proponer en las líneas anteriores, pareciera entonces que la única forma de romper con el ciclo de la violencia se encuentra en el pasado, no en el presente o en el futuro. Pero, entonces, ¿se puede cambiar el pasado?

\section{El miedo a la paz}

El conflicto en términos del pensamiento disímil, es decir, el que se relaciona con el pensar diferente, se encuentra encarnado frente al proceso de paz en el ex presidente y hoy senador Álvaro Uribe Vélez, como jefe de la oposición y principal enemigo del proceso de paz con las FARC-EP. ¿Cómo relata el pasado actualizado en presente?: 
Empezó el plan Colombia, el presidente Clinton, el presidente pastrana, 170.000 hectáreas de droga, bajo a 47.000, ahora 200.000, a los narcotraficantes mayores de las FARC, el primer grupo de cocaína del mundo, no los extraditan ni tienen sanción interna, les dan elegibilidad, responsables de todo tipo de delitos atroces, EEUU no les permitiría ser elegibles, ni tampoco EEUU los eximiría de una sanción adecuada. (...) Violación de niñas 6.800 mujeres violadas, 11.700 niños reclutados, que basta con reconocer, no hay cárcel, hay elegibilidad política, eso no es sanción adecuada, eso es amnistía. (...) La economía Colombia va mal porque se acabaron las condiciones de confianza de inversión, por el gasto público, $54 \%$ de endeudamiento, y ahora impuestos elevadísimos que han desterrado la inversión, y ahora más impuestos, expropiaciones acordadas con las FARC, todo eso puede afectar mas la economía, no hay petróleo sino para cinco años, gas para seis años, no ha habido exploración al ritmo que se requiere, esto puede llevar..., y 'por qué no la ha habido?, por narcotráfico y violencia de las mismas FARC, ¿̇esto puede llevar a qué? Esto puede llevar a una crisis económica, generadora de una gran crisis social que la ciudadanía diga: estamos cansados con Santos, elijamos a Timochenko (Uribe Vélez, 2016)

El pasado así actualizado al presente refuerza la construcción de ese ellos que se aleja del nosotros, pero además en ese proceso de paz, se asume la consolidación de la perdida de las formas vigentes del poder, a partir de la latencia del futuro, donde de hecho se llega a señalar cómo el comandante de las FARC-EP sería presidente del espacio Colombia. Es evidente, en este fragmento de la explicación de Uribe, cómo las cifras y las relaciones entre actos criminales y la economía se establecen en un proceso de producción de un pasado que no solo es imposible olvidar o reconstruir, sino que además se le señala como el desencadenador de problemas económicos y crisis sociales, que llevaron a que la democracia colombiana se pierda en las manos de esos otros que, a diferencia de ese nosotros que defiende el senador, si podrán llevar la democracia al futuro deseado.

Es entonces en el futuro deseado por ese nosotros donde se materializan formas de organización social, económica y política de una forma de orden social exclusiva, desconociendo los procesos de violencia del país en los cuales el mismo Estado colombiano ha sido señalado y reconocido como responsable por tribunales de justicia internacionales, como es el caso del genocidio de la Unión Patriótica. Así, el pasado, la historia, se utiliza con una finalidad específica, la consolidación del miedo al futu- 
ro, la latencia en este caso de «una crisis económica, generadora de una gran crisis social» (Uribe Vélez, 2016).

En el presente se planteó la pregunta ¿́se puede cambiar el pasado?, la respuesta es afirmativa, las narraciones del pasado y de la historia se pueden modificar. Con estas modificaciones también se puede mentir, en especial sobre el futuro, en los posibles mecanismos de actualización del pasado en el presente, siempre con finalidades específicas. El miedo a la paz se materializa, entonces, en los futuros posibles, que representan para aquellos que detentan el poder, en posibles cambios o giros que les represente perder sus beneficios o la acumulación de recursos diversos que garantizan sus formas de desarrollo.

Es entonces posible cambiar el pasado, actualizarlo en el presente. El problema finalmente se materializa no en la posibilidad de cambiarlo por medio de las narraciones que se configuran, sino en las apuestas que se generan en éstas, pues si se trabajan desde la posibilidad que brinda el encuentro con la diferencia y la esperanza, seguramente se podrá contar con posibilidades de futuro en las que el miedo se desvincule de la dicotomía del nosotros y el ellos, un futuro con otras latencias, donde el miedo se materialice en que no puedan seguir desapareciendo profesores de las universidades por pensar diferente, violando mujeres, reclutando niños, desplazando campesinos, en últimas, sentir miedo por lo que ya se vive en el presente y no seguir desplazando las acciones y prácticas al futuro.

\section{Referencias bibliográficas}

Arendt, H. (1997). ¿Qué es la politica? Barcelona, España: Paidós.

Cabra Ayala, N. A. y Aschner Restrepo, C. (2017). Saberes Nómadas: Derivas del pensamiento propio. Bogotá, Colombia: Universidad Central.

Camps, V. (2012). El gobierno de las emociones. Barcelona, España: Herder.

Castro Gómez, S. (16 de Mayo de 2017). Documental Los miedos a la paz. Medellín, F. y Gutierrez, F. (Entrevistadores).

Deleuze, G. (2001). Spinoza: Filosofía práctica. Barcelona, España: Tusquets.

Gómez Restrepo, C. (15 de Marzo de 2017). Documental Los miedos a la paz. (Gutiérrez, F. y Ruiz, J. (Entrevistadores). 
Gutierrez, P. (25 de Abril de 2017). Documental Los miedos a la paz. Ruiz, J. (Entrevistador).

Laclau, E. (2014). Los fundamentos retóricos de la sociedad. Buenos Aires, Argentina: Fondo de Cultura Económica.

Lakoff, G. y Johnson, M. (2017). Metáforas de la vida cotidiana. Madrid, España: Cátedra.

López, C. (4 de Abril de 2017). Documental los miedos a la paz. Documental Los miedos a la paz. Medellín, F. y Gutierrez, F. (Entrevistadores).

Martínez, C. E. (15 de Mayo de 2017). Documental Los miedos a la paz. Medellín, F. y Ruiz, J. (Entrevistadores).

Nussbaum, M. (2017). Paisaje de los pensamientos. Barcelona, España: Paidós.

Osses Rivera, S. L. y Suárez González, M. T. (2017). Lo que encubre la violencia: un estudio para seguir comprendiendo el conflicto en Colombia. En K. Kluster, K. Conflictividad y violencia en América Latina, pp. 191-228. México, México: UACM.

Osses, S. L. (10 de Septiembre de 2017). Documental Los miedos a la paz. Gutierrez, F. (Entrevistador).

PNUD. (2003). El conflicto, callejón con salida: informe nacional de Desarrollo Humano para Colombia. Bogotá, Colombia: PNUD.

Restrepo, E. (13 de Mayo de 2017). Documental Los miedos a la paz. (Gutierrez, F. Medellín, F. y Ruiz, J. (Entrevistadores)

Robin, C. (2009). El miedo. Historia de una idea política. México: Fondo de Cultura Económica.

Rorty, R. (2008). Feminismo, ideología y deconstrucción. En S. Zizek, Ideología: un mapa de la cuestión (págs. 253 - 262). Buenos Aires: Fondo de Cultura Económica.

Arendt, H. (1997). ¿Qué es la política? Barcelona: Paidós.

Cabra Ayala, N. A., \& Aschner Restrepo, C. (2017). Saberes Nómadas:

Derivas del pensamiento propio. Bogotá : Universidad Central.

Camps, V. (2012). El gobierno de las emociones. Barcelona: Herder.

Castro Gómez, S. (16 de Mayo de 2017). Documental los miedos a la paz. (F. Medellín, \& F. Gutierrez, Entrevistadores)

Deleuze, G. (2001). Spinoza: Filosofía práctica. Barcelona: Tusquets. 
Gómez Restrepo, C. (15 de Marzo de 2017). Documental los miedos a la paz. (F. Gutiérrez, \& J. Ruiz, Entrevistadores)

Gutierrez, P. (25 de Abril de 2017). Documental los miedos a la paz. (J. Ruiz, Entrevistador)

Laclau, E. (2014). Los fundamentos retóricos de la sociedad. Buenos Aires: Fondo de Cultura Económica.

Lakoff, G., \& Johnson, M. (2017). metaforas de la vida cotidiana. Madrid: Cátedra.

López, C. (4 de Abril de 2017). Documental los miedos a la paz. (F. Medellín, \& F. Gutiérrez, Entrevistadores)

Martínez, C. E. (15 de Mayo de 2017). Documental los miedos a la paz. (F. Medellín, \& J. C. Ruiz, Entrevistadores)

Nussbaum, M. (2017). Paisaje de los pensamientos. Barcelona: Paidós.

Osses Rivera, S. L., \& Suárez González, M. T. (2017). Lo que encubre la violencia: un estudio para seguir comprendiendo el conflicto en Colombia. En K. Kluster, Conflictividad y violencia en América Latina (págs. 191-228). México: UACM.

Osses, S. L. (10 de Septiembre de 2017). Documental los miedos a la paz. (F. Gutierrez, Entrevistador)

PNUD. (2003). El conflicto, callejón con salida: informe nacional de Desarrollo Humano para Colombia. Bogotá: PNUD.

Restrepo, E. (13 de Mayo de 2017). Documental los miedos a la paz. (F. Gutierrez, F. Medellín, \& J. C. Ruiz, Entrevistadores)

Robin, C. (2009). El miedo. Historia de una idea politica. México: Fondo de Cultura Económica.

Rorty, R. (2008). Feminismo, ideología y deconstrucción. En Zizek, S. Ideología: un mapa de la cuestión, pp. 253 - 262. Buenos Aires, Argentina: Fondo de Cultura Económica.

Suarez Gonzalez, M. T. (2017). Representaciones de justicia y paz en la ley 975 de 2005: aproximación discursiva y social (tesis doctoral). Universidad Pedagógica y Tecnológica de Colombia, Tunja, Colombia.

Torres Carrillo, A. (2013). El retorno a la comunidad: problemas, debates $y$ desafíos de vivir juntos. Bogotá, Colombia: CINDE el Búho.

Uribe Velez, A. (23 de Septiembre de 2016). Alvaro Uribe explica su postura sobre el Acuerdo de Paz. Velez, L. (Entrevistador). 
Useche, O. (5 de Mayo de 2017). Documental Los miedos a la paz. Medellín, F. y Gutiérrez, F. (Entrevistadores).

Wallerstein, I. (1996). Abrir las ciencias sociales. México, México: Siglo XXI.

Wallerstein, I. (1998). Impensar las ciencias sociales. México, México: Siglo XXI.

Wilde, O. (2016). El alma del hombre bajo el socialismo. Barcelona, España: El viejo topo.

Zuleta, E. (2015). Elgio a la dificultad y otros ensayos. Bogotá, Colombia: Ariel. 